\title{
The Overcontrol in Youth Checklist (OCYC): Behavioral and neural validation of a parent-report of child overcontrol in early childhood
}

\author{
Kirsten Gilbert ${ }^{\mathrm{a},{ }^{,}, \text {Deanna M. Barch }}{ }^{\mathrm{a}, \mathrm{b}, \mathrm{c}}$, Joan L. Luby ${ }^{\mathrm{a}}$ \\ aDepartment of Psychiatry, Washington University in St. Louis, 4444 Forest Park, Suite 2100, St. \\ Louis MO, 63108 USA \\ ${ }^{b}$ Department of Psychological \& Brain Sciences, Washington University in St. Louis, Box 1125, \\ One Brookings Drive, St. Louis, MO, 63130 USA \\ 'Department of Radiology, Washington University in St. Louis, Box 1125, One Brookings Drive, \\ St. Louis, MO, 63130 USA
}

\begin{abstract}
Self-control is protective against psychopathology in childhood. However, too much self-control, namely overcontrol, potentiates risk. Overcontrol is a constellation of child characteristics related to high need for control, perfectionism, inflexibility, social comparison, and performance monitoring and is a transdiagnostic risk factor associated with psychiatric disorders across the lifespan. However, there are no quick and developmentally appropriate screeners to identify overcontrol in early childhood, when overconrol purportedly becomes stable. The current study validated the Overcontrol in Youth Checklist (OCYC) in 4-7 year old children and examined relationships with cognitive, social, and psychiatric, neural and behavioral indicators. The OCYC demonstrated good psychometrics and was associated with deficits in cognitive shifting, social functioning, and preschool psychopathology. Higher OCYC scores were associated with a blunted $\triangle E R N$, an indicator of performance monitoring in preschoolers. Findings demonstrate the OCYC to be a developmentally valid measure of overcontrol that identifies this transdiagnostic risk factor early in development.
\end{abstract}

\footnotetext{
Terms of use and reuse: academic research for non-commercial purposes, see here for full terms. https://www.springer.com/aamterms-v1

*Corresponding Author: Kirsten Gilbert, PhD, Washington University School of Medicine, Department of Psychiatry, 4444 Forest Park, Suite 2100, St. Louis MO 63108, Phone: 314-747-0001, Fax: 314-286-2732, gilbertk@ wustl.edu.

Publisher's Disclaimer: This Author Accepted Manuscript is a PDF file of an unedited peer-reviewed manuscript that has been accepted for publication but has not been copyedited or corrected. The official version of record that is published in the journal is kept up to date and so may therefore differ from this version.

Conflict of Interest: KG, DB and JL have received research grants from National Institute of Health.

Research involving human participants and/or animals: All procedures performed in studies involving human participants were in accordance with the ethical standards of the institutional and/or national research committee and with the 1964 Helsinki declaration and its later amendments or comparable ethical standards.

Informed Consent: Informed consent was obtained from all individual participants included in the study.
} 


\section{Keywords}

overcontrol; early childhood; transdiagnostic; behavioral inhibition; error-related negativity

\section{Introduction}

Self-control develops rapidly in early childhood, is adaptive, and is protective against onset of psychopathology [1]. A lack of self-control, or undercontrol, is widely studied in children and contributes to externalizing presentations [2]. Conversely, excessive self-control, (or given self-control may not fully be developed in early childhood, excessive need for control), or 'overcontrol,' is also implicated across internalizing presentations of child psychopathology $[3,4]$, but has received much less research attention. Overcontrol is associated with multiple psychiatric disorders across the lifespan, including social anxiety disorder, obsessive-compulsive disorder (OCD), anorexia nervosa and depression [5-7], and thus appears to be a transdiagnostically relevant construct.

Overcontrol taps a desire for control, structure, perfection, and aversion to making mistakes. It appears to most commonly occur in the context of behavioral inhibition (BI), or the hesitancy to approach new and unfamiliar people [e.g., shyness; 8]. However, overcontrol is conceptually distinguishable from BI, demonstrating an independent developmental trajectory, as BI is identifiable in infants and toddlers, while overcontrol does not become stable until age 5 [3, 9]. BI has been extensively studied in young children [e.g., 10, 11], yet only a subset of these children will develop a psychiatric disorder [12]. Thus, characterizing the subset of children with BI who display elevated risk, potentially those exhibiting overcontrol, may be most clinically relevant. The purpose of the current study was to validate a short, easy to administer, parent-reported measure of overcontrol in young children to efficiently identify this transdiagnostic risk marker in early childhood.

Past work on overcontrol in youth comes from several independent lines of research, with the personality literature first to coin the term $[4,13]$. From this perspective, overcontrolled children are introverted and tense, emotionally sensitive, but also agreeable and prosocial $[14,15]$. This personality style has been replicated across cultures and ages, demonstrating stability across the lifespan $[16,17]$. Overcontrol can also be identified using the Five Factor Model of personality [FFM; 18]; overcontrolled youth demonstrate low extraversion and high neuroticism, with some work indicating low openness and elevated conscientiousness and agreeableness $[4,17,19]$.

The temperament literature conceptualizes overcontrol as a form of 'reactive overcontrol,' or rigid, inflexible, behavioral inhibition [3]. This literature characterizes overcontrolled children as shy and inhibited, exhibiting high concern with making mistakes (and as a result are often young perfectionists), displaying high inhibitory control but poor cognitive flexibility and shifting, and exhibiting anxious apprehension [3,20]. When exhibiting high negative emotionality, these children are emotionally expressive at young ages. However, over time, overcontrolled children learn to inhibit emotion expression and are prone to reactive withdrawal [20]. 
Overcontrol has recently been examined in the context of BI. In the context of elevated BI, overcontrol is studied as a moderating risk factor [11,21]. Although many overcontrolled children exhibit a behaviorally inhibited temperament, not all children with elevated BI are overcontrolled. In the BI literature, overcontrol is indexed using task-based inhibitory control [21] or a neural marker, the error-related negativity (ERN), an event-related potential (ERP). The ERN is associated with anxious apprehension, error and performance monitoring, and reactive control processing [see below for more on this indicator; 22, 23, 24].

\section{Outcomes related to overcontrol in children}

The constellation of characteristics that make up overcontrol in early childhood often result in poor social functioning, including social withdrawal, peer rejection, high conflict in parent-child relationships, and loneliness, which persist through adolescence and adulthood $[17,20,25,26]$. However, overcontrolled children are often prosocial and desire social interactions, so poor social functioning can manifest as high social concern combined with a lack of age appropriate social skills $[15,20]$.

Additionally, overcontrol is associated with high internalizing symptoms in youth [27]. In children with elevated BI, overcontrol potentiates risk for social anxiety [21, 28, 29]. Characteristics of overcontrol (e.g., high concern for errors, perfectionism, cognitive inflexibility) have been theorized to be an endophenotype conferring risk for OCD and anorexia nervosa $[6,30]$.

\section{Neural and behavioral indicators of performance monitoring and overcontrol}

Although most research examines overcontrol as a constellation of temperamental/ personality characteristics, the BI literature has utilized neural (ERN) and behavioral indicators of overcontrol that overlap with performance monitoring (NIMH Research Domain Criteria (RDoC) Cognitive control: Performance monitoring). Specifically, the ERN is a negative deflection occurring within 50ms of making a behavioral error and indexes non-conscious responding to errors [24]. A larger (more negative) ERN is evident in anxiety disorders, particularly OCD, a disorder characterized by heightened concern over errors [31], and a larger ERN predicts onset of anxiety disorders in school-aged children [32]. The ERN is associated with checking behaviors and self-monitoring [33] and has been equated with overcontrol [11].

There is some evidence the ERN shows developmental specificity in its relationship with fear and anxiety. Most literature demonstrates a larger ERN in relation to overcontrol and anxiety outcomes in older children and adults, however an emerging literature indicates the opposite relationship in young children. In 6-year-old children, temperamental fear, anxiety symptoms and children with maternal history of anxiety disorders are associated with a blunted ERN [34, 35]. Age 3 fear predicted a blunted ERN at age 6 [23]. Although it is unclear why the relationship between the ERN and anxiety flips developmentally, it has been theorized to be due to changing phenomenology of anxiety from external to internal sources and/or the differential development of the rostral versus dorsal ACC, from where the ERN is thought to emerge [23, 35]. 
Behavioral adaptations following errors also index performance monitoring and may be tapping into overcontrol. These include slowing down after making a mistake (post-error slowing), which could adaptively improve later performance (post-error accuracy) [36]. However, post-error slowing may also index behavioral interference due to focusing on the error [37, 38], leading to worse subsequent performance. Although no work has examined how these behavioral indicators are associated with overcontrol in youth, they may provide meaningful information regarding cognitive patterns associated with overcontrol.

\section{The current study}

Overcontrol is a purported early-emerging transdiagnostic risk factor contributing to psychopathology. Identifying this characteristic early in childhood could lead to targeted early interventions that prevent onset of psychopathology and promote adaptive psychological functioning across the lifespan. Moreover, mapping a quick behavioral measure onto neural functioning could help link dimensional behavioral constructs implicated in mental disorders with neurobiological systems. However, currently there are no quick, feasible, and developmentally appropriate screening tools to identify overcontrol in early childhood. Past research has either used clustering or modeling analytical techniques of multiple characteristics, task-based measures that only capture aspects of the characteristic, or neural markers, each of which has limitations as measurement approaches (long or resource intensive).

The current study had three aims, with the overarching goal of validating a developmentally appropriate screening measure of overcontrol for early childhood, when these characteristics first become stable around age five [3]. First, we developed a brief parent-report questionnaire assessing early childhood overcontrol and tested its psychometric properties. Second, we examined how this measure related to psychological functioning, including cognitive and social functioning, behavioral inhibition and activation, and psychopathology. Third, we investigated whether this measure mapped onto neurobiological (EEG) and behavioral functioning that indexes heightened performance monitoring and overcontrol, including the ERN, post-error slowing and post-error accuracy. We hypothesized that children exhibiting higher overcontrol would display deficits in cognitive shifting (but not other measures of executive functioning) and social functioning, and elevated BI and internalizing presentations. We did not expect relationships between the OCYC and behavioral activation (BAS) or externalizing presentations. We also hypothesized that overcontrol would be associated with a blunted ERN, increased post error slowing due to perseverative attention towards the error, and increased post-error accuracy as a compensatory behavior to decrease subsequent errors.

\section{Methods}

\section{Participants}

95 depressed preschoolers and 52 healthy children aged 3-7 years

$(\mathrm{N}=147 ; \mathrm{M}=5.29, \mathrm{SD}=1.02)$ and their caregivers participated. Preschool depression prevalence rates of 1\%-2\% are comparable to school-age depression[39], and depressed preschoolers were from a randomized clinical trial [PCIT-ED; Parent-Child Interaction 
Therapy Emotion Development;40]. The majority completed assessments at baseline $(42.2 \%)$, a subset (4.8\%) attended a separate session soon after baseline and some completed measures following therapy (22.4\%). Treatment targeted emotional development and did not address overcontrol. A comparison sample of community healthy preschoolers completed a one-time session, confirming mental health status with below clinical $T$-scores on the Child Behavior Checklist [41, 42] and matched on gender, age, ethnicity and SES. A subset of children completed the neural ERP assessment $(n=64)$; showing no demographic differences ( $p$ ' $>.05)$ compared to the larger sample. A separate subset of parents $(\mathrm{n}=55)$ completed a second assessment of overcontrol to examine test-retest reliability. The current sample was part of an exploratory study to identify overcontrol in early childhood. All preschoolers in ongoing studies were invited to participate. Given overcontrol exists on a continuum across clinical and healthy samples, it was ideal to include both clinically depressed and healthy preschoolers.

\section{Measures}

Overcontrol in Youth Checklist.-The overcontrol in youth checklist (OCYC) was designed to assess behavioral manifestations of overcontrol in young children. The OCYC began as a 25 -item parent-reported measure including original items and items developmentally adapted from currently validated questionnaires [including the Behavioral Inhibition Questionnaire; Child Retrospective Perfectionism Questionnaire, IowaNetherlands Comparison Orientation Measure, Child Yale-Brown Obsessive Compulsive Scale, and Child and Adolescent Perfectionism Scale; 43, 44-46]. The OCYC assesses early childhood inflexibility, perfectionism, anxious apprehension, checking and social comparison that contribute to overcontrol. Parents are asked Yes/No questions about how their child acts most of the time, using the previous year as a time anchor. Dichotomous responses (yes/no) were chosen to create a screening checklist that is highly feasible in clinical and research settings and only takes 2 to 4 minutes to complete. Full-scale validation below and Apendix.

The Behavior Rating Inventory for Executive Function-Preschool version [BRIEF-P; 47] and The Behavior Rating Inventory for Executive Function BRIEF; Gioia et al., 2000) The BRIEF-P ( $\mathrm{n}=86$ ) was completed by parents with children $<6$ years and the BRIEF $(\mathrm{n}=58)$ was completed by parents with children $>6$ years. The BRIEF/-P measure behavioral manifestations of executive function, scored on a 3-point scale from never, sometimes, and often. Scores are summed, age-normed $T$-scores are used, and higher scores indicate greater impairment. Overlapping subscales for the BRIEF-P and BRIEF include inhibitory control (Inhibition), cognitive and behavioral flexibility (Shifting), emotional regulation (Emotional control), working memory (Working memory) and ability to plan and organize cognition and problem-solving (Plan/organize).

The Behavioral Inhibition and Activation Scales-Revised.-The Behavioral Inhibition and Activation Scales (BIS/BAS) assess child's behavioral inhibition (hesitancy to approach novelty) and behavioral activation (appetitive and approach motives). The revised validated version for younger children includes BAS Drive, BAS Reward Responsiveness 
and BIS subscales [48]. Internal consistency was adequate in the current sample (BIS $a=.72$, BAS-drive $\mathrm{a}=.82$, BAS-reward $\mathrm{a}=.82$ ).

Income-to-needs ratio.-This ratio was a measure of socioeconomic status computed by dividing total family income by the federal poverty level, based on family size.

Kiddie Schedule for Affective Disorders, Early Childhood.-Preschool-onset major depression (PO-MDD) and anxiety (separation anxiety disorder, generalized anxiety disorder, social anxiety disorder) and externalizing (oppositional defiant disorder, conduct disorder, ADHD) disorders were determined using the Kiddie Schedule for Affective Disorders, Early Childhood Version (K-SADS-EC; Gaffrey, Luby 2012). Interviews were administered by masters level clinicians to the primary caregiver of children in the clinical trial. To have PO-MDD, caregivers reported their child experienced $\geq 4$ DSM criteria for major depression in the past month. All diagnoses underwent case consultation and reliability across diagnoses was good $(\kappa=.88)$.

MacArthur Health Behavior Questionnaire.-Parents of depressed preschoolers completed the Peer Relations subscale of the MacArthur Health and Behavior Questionnaire- Parent 1.0 for children aged 4-8 [49]. This subscale consists of the 8-item peer acceptance/rejection and the 3-item bullied by peers subscales. Parents report on their child's experiences from 1 (not at all like) to 4 (very much like). Internal consistency for peer relations, peer acceptance, and bullied were good ( $\alpha^{\prime} s=.89, .87, .77$, respectively).

Child Behavior Checklist.-All parents completed the Child Behavior Checklist (CBCL) [41, 42] appropriate for their child's age, a well-validated measure assessing internalizing and externalizing problems. Age-based and normed $T$-scores for symptombased subscales were used.

Go/No-go ERN task.-Children played The Zoo Game, a child-friendly go/no-go task [50]. Children were instructed to 'round up' escaped zoo animals by hitting a button (gotrials; $75 \%$ of trials), and told the orangutans were friends helping and should not be round up (no-go trials, $25 \%$ of trials). Children pressed a button as quickly as possible when they saw an animal on the screen (except orangutans). Animal stimuli were presented for $500 \mathrm{~ms}$, followed by a blank screen for $900 \mathrm{~ms}$ until the child responded; the intertrial interval was jittered 200-300ms. Children completed four blocks of 70 trials each with breaks between blocks. The task was presented on a 20-inch monitor using E-prime Software (Psychology Software Tools, Inc., Pittsburgh, PA; Schneider, Eschman \& Zuccolotto, 2002) and using a Logitech Gamepad F310 game controller. Prior to the task children were shown three boxes of prizes with increasingly attractive toys linked to differing points. Children were told every time they 'caught' an animal (except orangutans) they would accrue points to win a prize and with more points, they could pick a better prize.

Behavioral measures included reaction time (RT) and accuracy for error and correct trials. To measure behavioral slowing and accuracy on trials immediately following errors, posterror slowing (PES) and post-error accuracy (PEA) percentage change variables were created, which take into account individual differences in reaction time [37]. Reaction time 
or accuracy following a correct go-trial is subtracted from an incorrect no-go response, then divided by reaction time or accuracy following a correct go-trial [PES: (RT when prior trial was incorrect no-go - RT when prior trial was correct go)/RT when prior trial was correct go-trial] and [PEA: (Accuracy when prior trial was incorrect no-go - accuracy when prior trial was correct go)/accuracy when prior trial was correct go-trial].

\section{Procedure}

As part of the larger study, parents and children completed a series of behavioral, EEG, and therapy sessions. In the current study, parents completed measures about their child while the child completed the EEG. All study materials received IRB approval from Washington University in St. Louis, School of Medicine and consent and assent was obtained from all study participants prior to data collection.

After the study was described, children were seated in a chair $60 \mathrm{~cm}$ from the computer screen and EEG sensors were attached. Children first completed 24 total practice trials to ensure comprehension. The task took children approximately 15 minutes and parents were paid for their time and children received prizes. To examine stability of the OCYC, a subset of parents completed a second OCYC over 149.15 ( $\mathrm{SD}=19.98$; range: 92-198) days apart. Although a long duration, given the stability of this characteristic we felt it could be interpreted as test re-test marker of stability.

\section{Psychophysiological recording, processing, and analysis}

EEG was recorded using a BainVision ActiCHamp 32-channel electrode system (Brain Products, Germany), using a subset of the International 10/20 system sites and a ground electrode at FPz. Additional electrodes for electrooculogram (EOG) included 2 electrodes placed laterally to the eyes to record horizontal EOG and 2 electrodes placed above and below the left eye to detect blinks and eye movements to record vertical EOG. Continuously recorded EEG was digitized at $500 \mathrm{~Hz}$ with 24 bits of resolution and referenced to $\mathrm{Cz}$. Offline processing was performed using Brain Vision Analyzer software (Brain Products, Germany), including re-referencing to the average of TP9 and TP10 (adjacent to mastoids), and band-pass filtering from .1 to $30 \mathrm{~Hz}$. Eye blinks and movements were corrected using Gratton et al. [51] procdures. Physiological artifacts were rejected using a semi-automated procedure allowing maximum voltage steps of $50 \mu \mathrm{V}$, maximum absolute voltage difference of $175 \mu \mathrm{V}$ within $400 \mathrm{~ms}$, and minimum allowed activity of $.5 \mu \mathrm{V}$ within $100 \mathrm{~ms}$.

Response locked ERP's were averaged separately for correct and error trials with a baseline correction from -400 to $-200 \mathrm{~ms}$ before response. To measure the ERN, mean amplitude for the ERN and CRN were measured -100 to $100 \mathrm{~ms}$ prior to and following the response, based on visual inspection of grand averages. The $\triangle \mathrm{ERN}$ was calculated as the incorrect minus correct responses evaluated at $\mathrm{Cz}$, a commonly used location in ERN studies with preschool aged children $[23,35]$.

\section{Statistical analyses}

Psychometric evaluation of the OCYC included exploratory factor analysis to examine factor structure and determination of subscales, Chronbach's alpha and scale if item deleted, 
communalities to test internal consistency and Pearson's correlations to determine stability of OCYC scores. We tested demographic and group (PO-MDD versus healthy) differences on the OCYC to include as covariates. To test convergent validity, correlations and partial correlations, controlling for significant demographic and group differences, were completed between the OCYC and executive functioning (BRIEF), behavioral inhibition and activation (BIS/BAS), social functioning (HBQ) and dimensional symptoms of psychopathology (CBCL). We used independent samples $t$-tests to examine OCYC differences on comorbid anxiety and externalizing disorders in the PO-MDD group. We examined convergent validity with neurobiological and behavioral indicators of performance monitoring using correlations and partial correlations of OCYC and neural and task-based behavioral indicators. All statistical analyses were performed using SPSS (version 25).

\section{Results}

\section{Psychometric evaluation of OCYC}

Exploratory factor analyses of original 25 items $(n=147)$ using principal axis factoring with oblique rotation allowed for correlation between factors. Items were deleted in iterative models if they failed to sufficiently load on any factor or the item loaded in a theoretically uninterpretable fashion. This resulted in the retention of 21 items. In the final model, using direct oblimin rotation, Kaiser-Meyer-Olkin was .83 and Bartlett's test was significant, $(p<001)$, indicating suitable factorability of data and 3 components, explaining a cumulative $46.85 \%$ of the variance. All values demonstrated loadings $>0.4$ on only one factor with low loadings on other factors except two items $(7,25$; loadings $>0.3)$. These items were retained because Chronbach's a would have decreased if excluded; this is true for all included items (see Table 1). Visual inspection of scree plots validated the three-factor solution.

Factor 1 included 9 items tapping into inflexibility and frustration with change (e.g., "gets frustrated when s/he can't seem to get it right the first time..."). Factor 2 included 9 items regarding social concern and perfectionism (e.g., "frequently compares his/her abilities with that of peers and siblings"). Factor 3 included 3 items tapping BI (e.g., "is quiet and uncertain in new situations"). Internal consistency for each subscale was good ( $a$ 's $=0.86$, 0.80 , and 0.68 for Factors 1-3, respectively). The 21-item full scale demonstrated good internal consistency $(a=0.86)$ however, alpha increased $(a=0.88)$ when BI items were deleted. The inflexibility/frustration factor was correlated with social concern/perfectionism $(r=0.59, p<.001)$ but the BI subscale was not correlated with either subscale $\left(r^{\prime} s=.02, .01\right.$, $p$ 's $>.82$ inflexibility/frustration, social concern/perfectionism respectively). Given the moderate psychometric consistency, lack of association with other subscales, and availability of multiple existing valid measures of BI [52], we excluded the BI subscale from the measure, resulting in an 18 item total measure consisting of two 9-item subscales.

Test-retest reliability results indicated significant correlations with retest, inflexibility/ frustration: $r=.83$, social concern/perfectionism: $r=.65$, and total: $r=.77 ; p$ 's<.001, indicating stability of overcontrol across several months. Moreover, retest scores demonstrated good internal consistency: inflexibility/frustration: $\mathrm{a}=.82$, social concern/perfectionism: $\mathrm{a}=.77$ and total: $\mathrm{a}=.87$. 


\section{Preliminary results}

Older children exhibited higher total $(r=.17, p=.04)$ and social concern/perfectionism scores $(r=.23, p=.007)$. Income-to-needs, ( $p$ 's $>.16)$, race ( $p$ 's $>.17)$, or gender ( $p$ 's $>.39)$ were not associated with OCYC scores. Depressed preschoolers showed elevated inflexibility/ frustration $(t(145)=-11.98, p<001)$, social concern/perfectionism $(t(145)=-5.56 . p<.001)$ and total scores $(t(145)=-10.04, p<.001)$. As expected, BI (BIS; $\mathrm{M}=19.41, \mathrm{SD}=4.04)$ was associated with elevated inflexibility/frustration $(r=.23 . p=.007)$. social concern/ perfectionism ( $r=.57 . p<.001)$, and total scores $(r=.44, p<.001)$. Although overcontrol often occurs in the context of BI, in order to isolate the independent validity of overcontrol, all further analyses controlled for BI, age, and depression status.

\section{Convergent validity with executive and social functioning and temperament}

See Table 2 for partial correlations controlling for BI, age and depression status between OCYC scores with executive function (BRIEF), social functioning (HBQ), and behavioral activation (BAS). OCYC total scores were associated with deficits across executive functioning domains. Subscales demonstrated specificity as inflexibility/frustration was associated with BAS drive but not peer relations while social concern/perfectionism was associated with worse peer relations, and not BAS drive.

Because overcontrol was associated with impairments across executive functioning domains, we also utilized linear regressions predicting OCYC scores, controlling for BI, age and depression status, simultaneously entering all cognitive domains (inhibit, shift, emotional control, working memory, and plan/organize) to test which domains demonstrated incremental validity. Above covariates, only impairment in shifting predicted total $\left(F(5,117)=13.23, R=.85, \Delta R^{2}=.15, p<.001 ; B(\mathrm{SE})=.13(.03), \mathrm{p}<.001\right)$ and social concern/ perfectionism scores $\left(F(5,117)=3.42, R=.73, \Delta R^{2}=.07, p=.006 ; B(\mathrm{SE})=.05(.02), p=.02\right)$. Impairment in shifting $\left(F(5,117)=18.87, R=.87, \Delta R^{2}=.20, p<.001 ; B(\mathrm{SE})=.08(.02), p<.001\right)$ and inhibition $(B(\mathrm{SE})=.06(.02), p=.002)$ predicted inflexibility/frustration.

\section{Convergent validity with psychopathology}

Dimensional relationships between psychopathology and OCYC indicated when controlling for BI, age and depression, OCYC total scores were associated with higher symptom scores across all domains except anxiety (Table 2). Additionally, inflexibility/frustration was specific to elevated internalizing, depression, ADHD, and somatic symptoms. Because overcontrol was associated with impairments across most symptom domains, we again utilized linear regressions predicting OCYC total and subscale scores, controlling for BI, age and depression, simultaneously entering the externalizing and internalizing scores to test incremental validity. Externalizing symptoms significantly contributed to higher total $\left(F(2,121)=12.98, R=.81, \Delta R^{2}=.07, p<.001 ; B(\mathrm{SE})=.14(.03), \mathrm{p}<.001\right)$, inflexibility/frustration $\left(F(2,121)=15.30, R=.81, \Delta R^{2}=.09 . p<.001 ; B(\mathrm{SE})=.09(.02) . p<.001\right)$ and social comparison/ perfectionism scores $\left(F(2,121)=4.12, R=.51, \Delta R^{2}=.03, p=.03, B(\mathrm{SE})=.05(.02), p=.02\right)$.

Within the PO-MDD group, comorbid anxiety disorders $(\mathrm{n}=26 ; \mathrm{M}=5.53, \mathrm{SD}=2.94)$ were associated with a trend in elevated social concern/perfectionism $t(86)=-1.96 . p=.053$, compared to depressed preschoolers without an anxiety disorder $(\mathrm{M}=4.29, \mathrm{SD}=2.62)$ while 
comorbid externalizing disorders $(\mathrm{n}=27)$ were associated with elevated inflexibility/ frustration $(\mathrm{M}=7.41, \mathrm{SD}=1.72) t(86)=-3.28, p=.001)$ and total scores $(\mathrm{M}=12.63, \mathrm{SD}=3.54)$ $t(86)=-2.54, p=.01)$, compared with depressed preschoolers without an externalizing disorder (inflexibility: $\mathrm{M}=5.74, \mathrm{SD}=2.38$; total: $\mathrm{M}=10.15, \mathrm{SD}=4.49$ ).

\section{Neural and behavioral validity}

Behavioral and ERP results: Accuracy and RT data are in Table 3. RT differed as a function of response type, $F(1,63)=90.61, p<.001$, children were faster on error than correct trials. Post-error RT differed as a function of prior error response; children were slower on go-trials after a no-go error $(\mathrm{M}=633.83, \mathrm{SD}=161.75)$ compared with go-trials after a no-go correct response $(\mathrm{M}=538.41, \mathrm{SD}=108.63), F(1,63)=44.91 . p<.001$. PES and PEA were not significantly associated $r=-.15, p=.25$. Grand average response-locked ERP at $\mathrm{Cz}$ is depicted in Figure 1; the ERN response was more negative after errors than correct responses, $F(1,63)=112.55, p<.001$.

Associations with OCYC: Total and social concern/perfectionism scores demonstrated similar correlations with behavioral indicators: children with higher scores had faster RT's and more errors on no-go trials (see Table 3). Interestingly, elevated total and social concern/ perfectionism scores were positively associated with PEA: after making an error on a no-go trial, children with higher overcontrol were more likely to be correct on the following trial. For neural indicators, the total and both subscale scores were associated with a blunted $\triangle \mathrm{ERN}$. However, when controlling for $\mathrm{BI}$, age, and depression status, neural and behavioral findings were no longer significant.

\section{Discussion}

The current study provided initial psychometric validation of a novel, quick, and easy-toadminister parent-report measure of early childhood overcontrol, a risk factor associated with transdiagnostic psychopathology [5-7]. The OCYC, the first parent-report measure of childhood overcontrolled tendencies, demonstrated preliminary face validity and convergent validity with cognitive, social, psychopathological, performance-based behavioral and neural indicators in children aged 4-7 years old.

The OCYC is devised of a total and two subscales (inflexibility/frustration with change and social concern/perfectionism), all which demonstrated good internal consistency and testretest reliability. Convergent validity was evidenced by overcontrol being associated with elevated BI, and independent of demographic factors and BI, was associated with poor social functioning, including worse peer relations and being bullied and worse executive functioning. Notably, poor cognitive shifting/flexibility was the only executive functioning deficit to be incrementally associated over BI with all OCYC scales.

Psychopathological outcomes were also associated with overcontrol. Depressed preschoolers demonstrated higher overcontrol than healthy preschoolers, and within the depressed preschool sample, comorbid anxiety disorders were associated with trending elevated social concern/perfectionism while comorbid externalizing disorders were associated with elevated 
inflexibility/frustration with change and total scores. Moreover, dimensional externalizing symptoms predicted elevated overcontrol above BI, covariates and internalizing symptoms.

Although the association with externalizing presentations was not hypothesized, overcontrol stabilizes around ages 4-7 [3]. As such, the desire or need for control may be present while the cognitive ability to enact control may still be developing. In response to an inability to exert desired levels of control, externalizing outbursts and presentations might ensue. This speculative conclusion parallels the development of anxiety. Specifically, irritability and emotional outbursts often occur in the context of elevated anxiety in young children [53, 54]. Further support for this hypothesis is provided by a study demonstrating that 6 -year old children with externalizing symptoms as well as five specific symptoms (irritability, blaming others, not being liked by others, crying often and being solitary in early childhood) longitudinally predict anxiety in adolescence [55]. Of note, these five symptoms overlap with overcontrol in early childhood, possibly indicating an overlap between overcontrol and externalizing presentations at young ages that transitions into anxiety presentations across development. Future work would benefit from tracking the developmental trajectories and differing phenotypic presentations of overcontrol across development.

Overcontrol was associated with several indicators of behavioral performance: faster reaction times, possibly demonstrating increased engagement with the performance-focused task, yet also more errors. Following errors, overcontrolled children showed improved accuracy on the next trial. Previous work in adults indicates perfectionists demonstrate better post-error accuracy [56]. For overcontrolled children in the current study, the saliency of the error possibly increased attention to avoid a subsequent error on the next trial. Both the ERN and post-error behavioral adjustments are units of analysis in the Research Domain Criteria (RDoC) construct of performance monitoring and the OCYC was associated with both units, indicating it may represent a valid developmentally appropriate measure of performance monitoring in early childhood.

Importantly, the OCYC was also associated with a neural indicator, a blunted $\triangle \mathrm{ERN}$, consistent with research demonstrating temperamental fear, maternal history of anxiety [35] and anxiety symptoms [34] to be associated with a blunted $\triangle E R N$ in preschoolers. The $\triangle \mathrm{ERN}$ is thought to index error monitoring [22,24], and maps onto checking behaviors and social performance [33,57], all of which characterize overcontrol. Thus, the $\triangle \mathrm{ERN}$ may be a particularly useful neural marker for childhood overcontrol. However, findings did not hold after controlling for age, BI and depression status, suggesting larger samples will be needed to further investigate these potential interactive relationships.

Overcontrol is often studied within the context of BI, however current findings underscore that it is a dissociable construct that should be investigated independently. When controlling for BI (with exception of neural and behavioral markers), social and psychopathological impairments remained significant. Additionally, BI does not involve a cognitive component, while overcontrol demonstrated significant associations with executive functioning deficits, further supporting overcontrol to be a unique construct. 
Overcontrol is evident across the lifespan and measures for adolescents and adults similarly tap into the construct of overcontrol. The Childhood Retrospective Perfectionism Questionnaire (CHIRP) [45] has been adapted to children and adolescents age 7- 17 [58] and assesses perfectionism and preoccupation with details while the Pathological Obsessive Compulsive Personality Scale (POPS)[59] assesses emotional overcontrol, rigidity and perfectionism in adults. The current study developmentally extends the assessment of overcontrol and related constructs downward by validating the first parent-reported assessment in early childhood.

Multiple limitations should be considered. First, the sample size was small, and only a subset completed neural and behavioral measures. Moreover, the sample consisted of a dichotomous split between depressed and healthy preschoolers. Future studies should examine the OCYC in larger community samples, possibly enriched for BI, to understand dimensional overcontrol from normative to pathological. Second, psychometric validation should be replicated across broader age ranges to further examine development and stability of overcontrol. Third, neural findings did not hold after controlling for age, BI and depression status. Given there is immense change in the ERN between the ages of 3 to 8 [23], development could be influencing findings. It will be imperative for future work to explore developmental trajectories of overcontrol to parse interactive roles with BI and development on neural and behavioral indicators.

\section{Summary}

Overcontrol is an early-emerging transdiagnostic characteristic associated with psychopathology and poor outcomes. The current study demonstrates overcontrol can be detected in early childhood using the OCYC. The OCYC showed face validity, convergent validity, and some discriminant validity from BI, suggesting it is a developmentally valid construct in young children. Given the importance of early intervention, the OCYC may aid in identifying this transdiagnostic risk factor early in development, prior to psychopathology.

\section{Supplementary Material}

Refer to Web version on PubMed Central for supplementary material.

\section{Acknowledgements:}

The authors wish to thank the many parents and children who participated in the Parent-Child Interaction Treatment Emotion Development (PCIT-ED) study.

Funding: This work was funded by the National Institute of Health (R01MH098454-04; ClinicalTrials.gov identifier: NCT02076425; K23 MH115074-01).

\section{References}

1. Moffitt TE, et al., A gradient of childhood self-control predicts health, wealth and public safety. Proc Natl Acad Sci U S A, 2011 108: p. 2693-2698. [PubMed: 21262822]

2. Eisenberg N, et al., The relations of regulation and emotionality to childrens externalizing and internalizing problem behavior. Child Development, 2001 72(4): p. 1112-1134. [PubMed: 11480937] 
3. Eisenberg N, Spinrad TL, and Eggum ND, Emotion-related self-regulation and its relation to children's maladjustment. Annual Review of Clinical Psychology, 2010 6: p. 495-525.

4. Donnellan BM and Robins RW, Resilient, overcontrolled and undercontrolled personality types: Issues and controversies. Social and Personality Psychology Compass, 2010 3: p. 1-14.

5. Zucker NL, et al., Anorexia nervosa and autism spectrum disorders: guided investigation of social cognitive endophenotypes. Psychol Bull, 2007 133(6): p. 976-1006. [PubMed: 17967091]

6. Altman SE and Shankman SA, What is the association between obsessive-compulsive disorder and eating disorders? Clin Psychol Rev, 2009 29(7): p. 638-46. [PubMed: 19744759]

7. Lynch TR, Radically Open dialectical behavior therapy: Theory and practice for treating disorders of overcontrol. 2018, Oakland, CA: Context Press.

8. Kagan J, et al., Behavioral inhibition to the unfamiliar. Child Development, 1984 55: p. 2212-2225.

9. Derryberry D and Rothbart MK, Reactive and effortful processes in the organization of temperament. Development \& Psychopathology, 1997 9(4): p. 633-52. [PubMed: 9448999]

10. Fox NA, et al., Behavioral inhibition: Linking biology and behavior within a developmental framework. Annu Rev Psychol, 2005 56: p. 235-62. [PubMed: 15709935]

11. Henderson HA, Pine DS, and Fox NA, Behavioral inhibition and developmental risk: a dualprocessing perspective. Neuropsychopharmacology, 2015 40(1): p. 207-24. [PubMed: 25065499]

12. Fox NA, et al., Commentary: To intervene or not? Appreciating or treating individual differences in childhood temperament--remarks on Rapee (2013). J Child Psychol Psychiatry, 2013 54(7): p. 789-90. [PubMed: 23746203]

13. Block JH and Block J, The role of ego-control and ego-resiliency in the organization of behavior. Development of cognition, affect and social relations: The Minnesota symposia on child psychology, 1980 13: p. 39-101.

14. Asendorpf JB and van Aken MA, Resilient, overcontrolled, and undercontrolled personality prototypes in childhood: Replicability, predictive power, and the trait-type issue. Journal of Personality and Social Psychology, 1999 77: p. 815-832. [PubMed: 10531673]

15. Robins RW, John OP, and Caspi A, The typological approach to studying personality, in Methods and models for studying the individual, Cairns RB, Bergman LR, and Kagan J, Editors. 1998, Sage Publications: Thousand Oaks, CA p. 135-160.

16. Hart D, et al., Personality and development in childhood: A person-centered approach. Monographs of the Society for Research in Child Development, 2003 68: p. i-122. [PubMed: 12875200]

17. Asendorpf JB, et al., Carving personality description at its joints: Confirmation of three replicable personality prototypes for both children and adults. European Journal of Personality, 2001 15(3): p. $169-198$.

18. Costa PT and McCrae RR, Professional Manaul: Revised NEO Personality Inventory (NEO-PI-R) and NEO Five-Factor Inventory (NEO-FFI). 1992, Odessa, FL: Psychological Assessment Resources.

19. Gilbert KE, et al., Identifying observed preschool personality types and their associations with longitudinal symptom trajectories. under review.

20. Eisenberg N, et al., Dispositional emotionality and regulation: Their role in predicting quality of social functioning. Journal of Personality and Social Psychology, 2000 78: p. 136-157. [PubMed: 10653511]

21. White LK, et al., Behavioral inhibition and anxiety: the moderating roles of inhibitory control and attention shifting. J Abnorm Child Psychol, 2011 39(5): p. 735-47. [PubMed: 21301953]

22. Moser JS, et al., On the relationship between anxiety and error monitoring: a meta analysis and conceptual framework. Frontiers in Human Neuroscience, 2013 7: p. 466. [PubMed: 23966928]

23. Meyer A, et al., Early temperamental fearfulness and the developmental trajectory of error-related brain activity. Dev Psychobiol, 2018 60(2): p. 224-231. [PubMed: 29344944]

24. Gehring WJ, et al., The error-related negativity (ERN/Ne), in The Oxford Handbook of EventRelated Potential Components, Luck SJ and Kappenman ES, Editors. 2012, Oxford University Press: New York p. 231-291. 
25. Eisenberg N and Fabes RA, Emotion, regulation, and the development of social competence, in Review of personality and social psychology, Clark MS, Editor. 1992, Sage: Newbury Park, CA. p. 119-150.

26. Newman DL, et al., Antecedents of adult interpersonal functioning: Effects of individual differences in age 3 temperament. Developmental Psychology, 1997 33: p. 206-217. [PubMed: 9147830]

27. Robins RW, et al., Resilient, overcontrolled, and undercontrolled boys: Three replicable personality types. Journal of Personality and Social Psychology, 1996 70(1): p. 157-171. [PubMed: 8558407]

28. Thorell L, Bohlin G, and Rydell A-M, Two types of inhibitory control: Predictive relations to social functioning. International Journal of Behavioral Development, 2004 28: p. 193-203.

29. Brooker RJ, Kiel EJ, and Buss KA, Early social fear predicts kindergarteners' socially anxious behaviors: Direct associations, moderation by inhibitory control, and differences from nonsocial fear. Emotion, 2016 16(7): p. 997-1010. [PubMed: 27213729]

30. Riesel A, et al., Overactive performance monitoring as an endophenotype for obsessive-compulsive disorder: Evidence from a treatment study. American Journal of Psychiatry, 2015 172(7): p. 66573. [PubMed: 25783756]

31. Gehring WJ, Himle J, and Nisenson LG, Action-monitoring dysfunction in obsessive-compulsive disorder. Psychological Science, 2000 11: p. 1-6. [PubMed: 11228836]

32. Meyer A, et al., Enhanced error-related brain activity in children predicts the onset of anxiety disorders between the ages of 6 and 9. J Abnorm Psychol, 2015 124(2): p. 266-74. [PubMed: 25643204]

33. Weinberg A, et al., Error-related negativity (ERN) and sustained threat: Conceptual framework and empirical evaluation in an adolescent sample. Psychophysiology, 2016 53(3): p. 372-85. [PubMed: 26877129]

34. Meyer A, et al., The development of the error-related negativity (ERN) and its relationship with anxiety: evidence from 8 to 13 year-olds. Dev Cogn Neurosci, 2012 2(1): p. 152-61. [PubMed: 22308177]

35. Torpey DC, et al., Error-related brain activity in young children: associations with parental anxiety and child temperamental negative emotionality. J Child Psychol Psychiatry, 2013 54(8): p. 854-62. [PubMed: 23294040]

36. Botvinick M, et al., Conflict monitoring and cognitive control. Psychological Review, 2001 108(3): p. 624-652. [PubMed: 11488380]

37. Buzzell GA, et al., Error-Induced Blindness: Error Detection Leads to Impaired Sensory Processing and Lower Accuracy at Short Response-Stimulus Intervals. J Neurosci, 2017 37(11): p. 2895-2903. [PubMed: 28193697]

38. Jentzsch I and Dudschig C, Why do we slow down after an error? Mechanisms underlying the effects of posterror slowing. Q J Exp Psychol (Hove), 2009 62(2): p. 209-18. [PubMed: 18720281]

39. Egger HL and Angold A, Common emotional and behavioral disorders in preschool children: presentation, nosology, and epidemiology. Journal of Child Psychology and Psychiatry, 2006 47(3-4): p. 313-337. [PubMed: 16492262]

40. Luby JL, et al., A randomized control trial of parent-child psychotherapy targeting emotion development for early childhood depression. American Journal of Psychiatiy, 2018.

41. Achenbach TM and Rescorla LA, Manual for the ASEBA preschool forms and profiles: An integrated system of multi-informant assessment; Child Behavior Checklist for ages 1 1/2 - 5; Language Development Survey; Caregiver-Teacher Report Form. 2000, University of Vermont.

42. Achenbach TM, Manual for ABES A School-Age Forms \& Profiles. 2001, Burlington, VT: Research Center for Children, Youth, \& Families, University of Vermont.

43. Kim J, et al., Psychometric properties of the Bbehavioral inhibition questionnaire in preschool children. Journal of Personality Assessment, 2011 93(6): p. 545-555. [PubMed: 21999378]

44. Scahill L, et al., Children's Yale-Brown Obsessive Compulsive Scale: Reliability and Validity. Journal of the American Academy of Child \& Adolescent Psychiatiy, 1997 36(6): p. 844-852. 
45. Southgate L, et al., The development of the childhood retrospective perfectionism questionnaire (CHIRP) in an eating disorder sample. Eur Eat Disord Rev, 200816(6): p. 451-62. [PubMed: 18444228]

46. Gibbons FX and Buunk BP, Individual differences in social comparison: Development of a scale of social comparison orientation. Journal of Personality and Social Psychology, 1999 76(1): p. 129142. [PubMed: 9972558]

47. Gioia GA, Espy KA, and Isquith PK, Behavior Rating Inventory of Executive Function-Preschool Version. 2003, Lutz, FL: Psychological Assessment Resources.

48. Pagliaccio D, et al., Revising the BIS/BAS scale to study development: Measurement invariance and normative effects of age and sex from childhood through adulthood. Psychological Assessment, 2015 28(4): p. 429-442. [PubMed: 26302106]

49. Armstrong JM, Goldstein LH, and g.o.O TMW. Assessment, Manual for the MacArthur Health and Behavior Questionnaire (HBQ 1.0), C MacArthur Foundation Research Network on Psychopathology and Development (Kupfer David J., Editor. 2003: University of Pittsburgh.

50. Grammer JK, et al., Age-related changes in error processing in young children: a school-based investigation. Developmental Cognitive Neuroscience, 2014 9: p. 93-105. [PubMed: 24631799]

51. Gratton G, Coles MG, and Donchin E, A new method for off-line removal of ocular artifact. Electroencephalography and Clinical Neurophysiology, 1983 55(4): p. 468-484. [PubMed: 6187540]

52. Bishop G, Spence SH, and Mcdonald C, Can parents and teachers provide a reliable and valid report of behavioral inhibition. Child Development, 2003 74(6): p. 1899-1917. [PubMed: 14669903]

53. Cornacchio D, et al., Irritability and anxiety severity among youth with anxiety. Journal of American Academy of Child and Adolescent Psychiatry, 2016 55(1): p. 54-61.

54. Stoddard J, et al., Irritability in child and adolescent anxiety disorders. Depression and Anxiety, 2014 31(7): p. 566-573. [PubMed: 23818321]

55. Rouquette A, et al., Emotional and Behavioral Symptom Network Structure in Elementary School Girls and Association With Anxiety Disorders and Depression in Adolescence and Early Adulthood: A Network Analysis. JAMA Psychiatry, 2018.

56. Stahl J, et al., Perfect error processing: Perfectionism-related variations in action monitoring and error processing mechanisms. Int J Psychophysiol, 2015 97(2): p. 153-62. [PubMed: 26071226]

57. Buzzell GA, et al., A Neurobehavioral Mechanism Linking Behaviorally Inhibited Temperament and Later Adolescent Social Anxiety. J Am Acad Child Adolesc Psychiatry, 2017 56(12): p. 10971105. [PubMed: 29173744]

58. Park JM, et al., Obsessive-compulsive personality traits in youth with obsessive-compulsive disorder. Child Psychiatry and Human Development, 2016 47: p. 281-290. [PubMed: 26160348]

59. Pinto A, Ansell EB, and Wright AGC. A new approach to the assessment of obsessive compulsive personality. in Integrated paper session conducted at the annual meeting of the Society for Personality Assessment 2011 Cambridge, MA. 


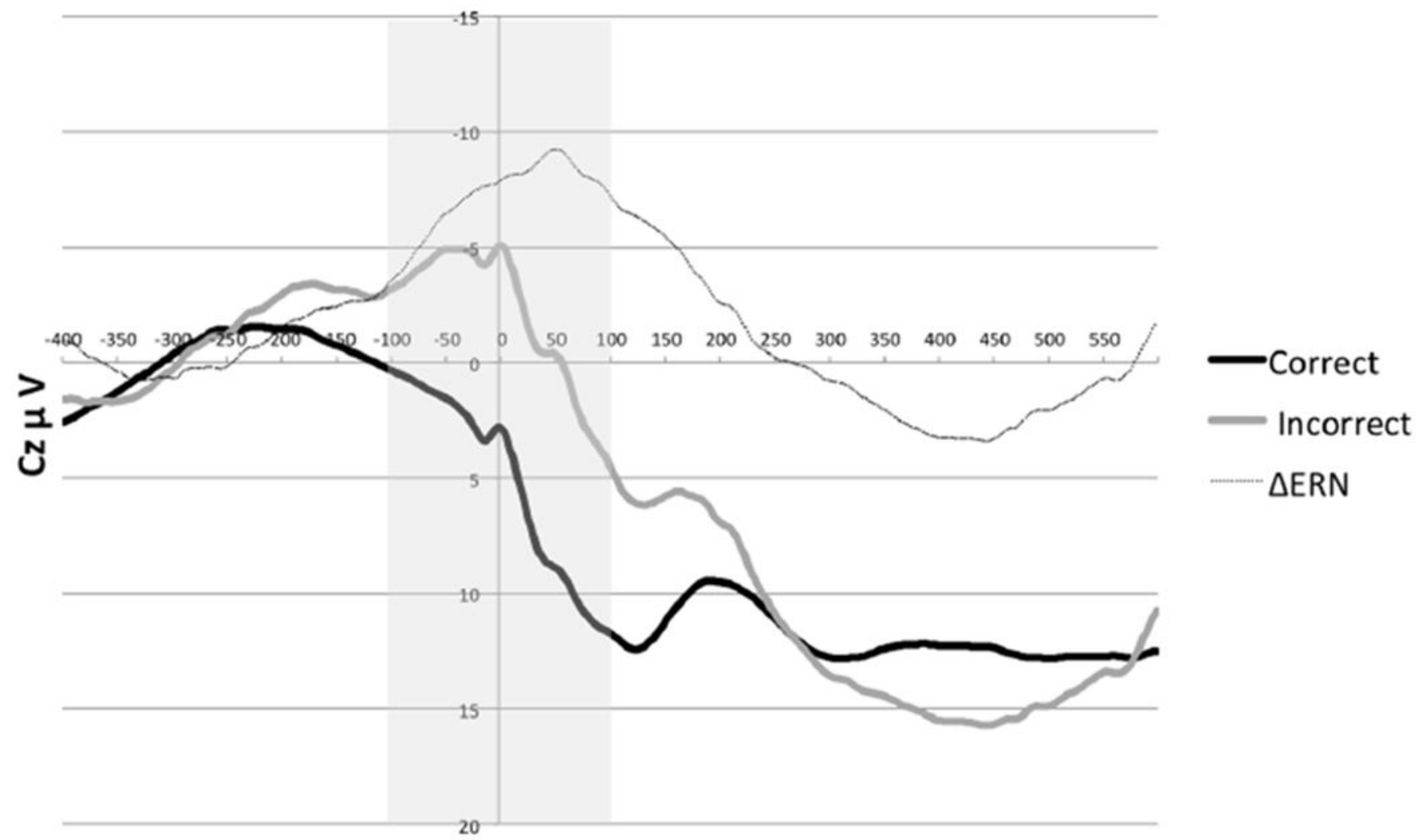

Figure 1.

Grand average response-locked ERPs at $\mathrm{Cz}$

Note: Electrode $\mathrm{Cz}$ is shown and indicates the time window (in gray) used to isolate the ERN 
Table 1.

Pattern Matrix and Communalities for PCA of 21-item OCYC.

\begin{tabular}{|c|c|c|c|c|c|}
\hline \multirow[b]{2}{*}{ Item } & \multicolumn{3}{|c|}{ Factors } & \multirow[b]{2}{*}{$\mathbf{r}$} & \multirow[b]{2}{*}{ Communalities } \\
\hline & $\mathbf{I}$ & II & III & & \\
\hline \multicolumn{6}{|l|}{ Factor I: Inflexibility/Frustration (Eigenvalue $=6.19,29.46 \%$ variance explained) } \\
\hline 4. Gets upset or has trouble when plans change at short notice & .727 & -.082 & .084 & .601 & .486 \\
\hline 20. Is easy-going and flexible $(\mathrm{R})$ & .663 & -.062 & .068 & .563 & .409 \\
\hline $\begin{array}{l}\text { 2. Finds schedule changes more difficult than peers (e.g., difficulty adjusting to school } \\
\text { transition, changes at home or in the family) }\end{array}$ & .635 & -.014 & .201 & .563 & .444 \\
\hline 16. Becomes upset if things don't go how s/he thinks they should & .616 & .019 & -.044 & .567 & .393 \\
\hline 19. Becomes very upset if criticized or given suggestions on how to do things differently & .564 & .271 & -.086 & .682 & .558 \\
\hline $\begin{array}{l}\text { 3. Gets frustrated when s/he can't seem to get it right the first time (e.g., when solving a } \\
\text { puzzle, when completing a math problem) }\end{array}$ & .550 & .052 & -.189 & .528 & .365 \\
\hline 23. Is rigid in his/her way of doing things & .539 & .196 & .049 & .587 & .441 \\
\hline $\begin{array}{l}\text { 14. Wants things done his/her own way (i.e., wants to play his/her way, wants to make the } \\
\text { rules) }\end{array}$ & .531 & .162 & -.191 & .577 & .430 \\
\hline 8. Has difficulty taking suggestions or receiving help from parents or peers & .487 & .174 & -.103 & .559 & .365 \\
\hline \multicolumn{6}{|l|}{ Factor II: Social concern/Perfectionism (Eigenvalue $=1.97,9.37 \%$ variance explained) } \\
\hline 6. Frequently compares his/her abilities with that of peers and siblings & -.20 & .713 & -.03 & .482 & .403 \\
\hline 17. Pays a lot of attention to how s/he does things compared with others & -.131 & .701 & -.029 & .551 & .425 \\
\hline 21. Gets mad at him/herself when s/he makes a mistake, especially in front of others & .153 & .620 & .083 & .598 & .507 \\
\hline 18. Becomes upset when s/he thinks s/he has done poorly on something & .099 & .467 & -.009 & .504 & .227 \\
\hline 25. Often feels not good enough compared with peers or siblings & .167 & .458 & .034 & .474 & .317 \\
\hline $\begin{array}{l}\text { 15. Looks for reassurance that s/he has done well or done the right thing more than } \\
\text { siblings or other kids his/her age }\end{array}$ & .087 & .440 & .077 & .423 & .244 \\
\hline $\begin{array}{l}\text { 10. Is concerned with doing things just right (e.g., hair parted the right way, food on plate } \\
\text { certain way) }\end{array}$ & .170 & .407 & .037 & .482 & .266 \\
\hline 7. Often thinks there is only one right way to do things & .283 & .388 & -.009 & .478 & .345 \\
\hline 24. Becomes upset when others don't follow the rules & .146 & .316 & .036 & .375 & .169 \\
\hline \multicolumn{6}{|l|}{ Factor IIII: Behavioral inhibition (Eigenvalue $=1.68,8.01 \%$ variance explained) } \\
\hline 13. Is quiet and uncertain in new situations & .123 & .060 & .678 & .489 & .487 \\
\hline 1. Is shy/hesitant when meeting new children & .021 & .026 & .671 & .560 & .450 \\
\hline 22. Likes being the center of attention $(\mathrm{R})$ & -.142 & -.007 & .510 & .365 & .277 \\
\hline
\end{tabular}

Note: Items 5, 9, 11, and 12 were deleted prior to final analysis; major loadings for each item are bolded; $\mathrm{r}=$ Corrected correlation between the item and its' subscale. 
Table 2.

Partial correlations (controlling for behavioral inhibition, age and depression) of OCYC and criterion variables

\begin{tabular}{|c|c|c|c|c|c|}
\hline & & & & \multicolumn{2}{|c|}{ OCYC } \\
\hline & Mean & SD & Total & Inflexibility/Frustration & Social Concern/Perfectionism \\
\hline \multicolumn{6}{|l|}{ OCYC } \\
\hline Total & 8.52 & 5.00 & -- & $.85^{* *}$ & $.83^{* * *}$ \\
\hline Inflexibility/frustration & 4.75 & 2.97 & & -- & $.42^{* *}$ \\
\hline Social concern/perfectionism & 3.76 & 2.63 & & & -- \\
\hline \multicolumn{6}{|l|}{ BRIEF } \\
\hline Inhibit & 56.15 & 12.90 & $.41^{* * *}$ & $.50^{* *}$ & $.19^{*}$ \\
\hline Shift & 57.11 & 13.55 & $.49^{* *}$ & $.54^{* * *}$ & $.27^{* * *}$ \\
\hline Emotional Control & 60.08 & 15.59 & $.44^{* *}$ & $.50^{* * *}$ & $.23^{*}$ \\
\hline Working Memory & 54.71 & 12.56 & $.32^{* *}$ & $.33^{* * *}$ & $.21^{*}$ \\
\hline Plan/Organize & 52.54 & 11.56 & $.43^{* * *}$ & $.43^{* * *}$ & $.29^{*}$ \\
\hline \multicolumn{6}{|l|}{$\mathrm{HBQ}^{a}$} \\
\hline Global Peer Relations & 3.43 & 0.48 & -.19 & -.05 & $-.29^{*}$ \\
\hline Peer Acceptance & 3.42 & 0.51 & -.20 & -.09 & $-.26^{*}$ \\
\hline Bullied & 1.56 & 0.56 & .15 & .001 & $.26^{*}$ \\
\hline \multicolumn{6}{|l|}{ BAS } \\
\hline Drive & 19.96 & 4.87 & $.23^{*}$ & $.31^{* * *}$ & .07 \\
\hline Reward Seeking & 23.75 & 3.05 & -.06 & -.02 & .09 \\
\hline \multicolumn{6}{|l|}{ CBCL } \\
\hline Internalizing & 54.93 & 12.21 & $.22^{*}$ & $.24^{*}$ & .13 \\
\hline Externalizing & 54.26 & 14.21 & $.46^{* *}$ & $.49^{* * *}$ & $.28^{*}$ \\
\hline Depression & 59.27 & 9.04 & $.20 *$ & $.23^{*}$ & .11 \\
\hline Anxiety & 58.17 & 9.72 & .07 & -.001 & .12 \\
\hline ADHD & 55.20 & 7.23 & $.19^{*}$ & $.26^{*}$ & .05 \\
\hline ODD & 60.81 & 10.85 & $.38^{* *}$ & $.44^{* * *}$ & $.21^{*}$ \\
\hline Somatic complaints & 54.80 & 6.44 & $.18^{*}$ & $.18^{*}$ & .12 \\
\hline
\end{tabular}

Note: BRIEF= Behavior Rating Inventory for Executive Functioning; HBQ= Health and Behavior Questionnaire; BAS= Behavioral Activation Revised scales; $\mathrm{CBCL}=$ Child Behavior Checklist.

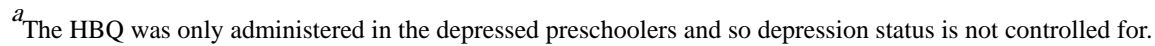

* $p<.05$

** $p<.001$ 


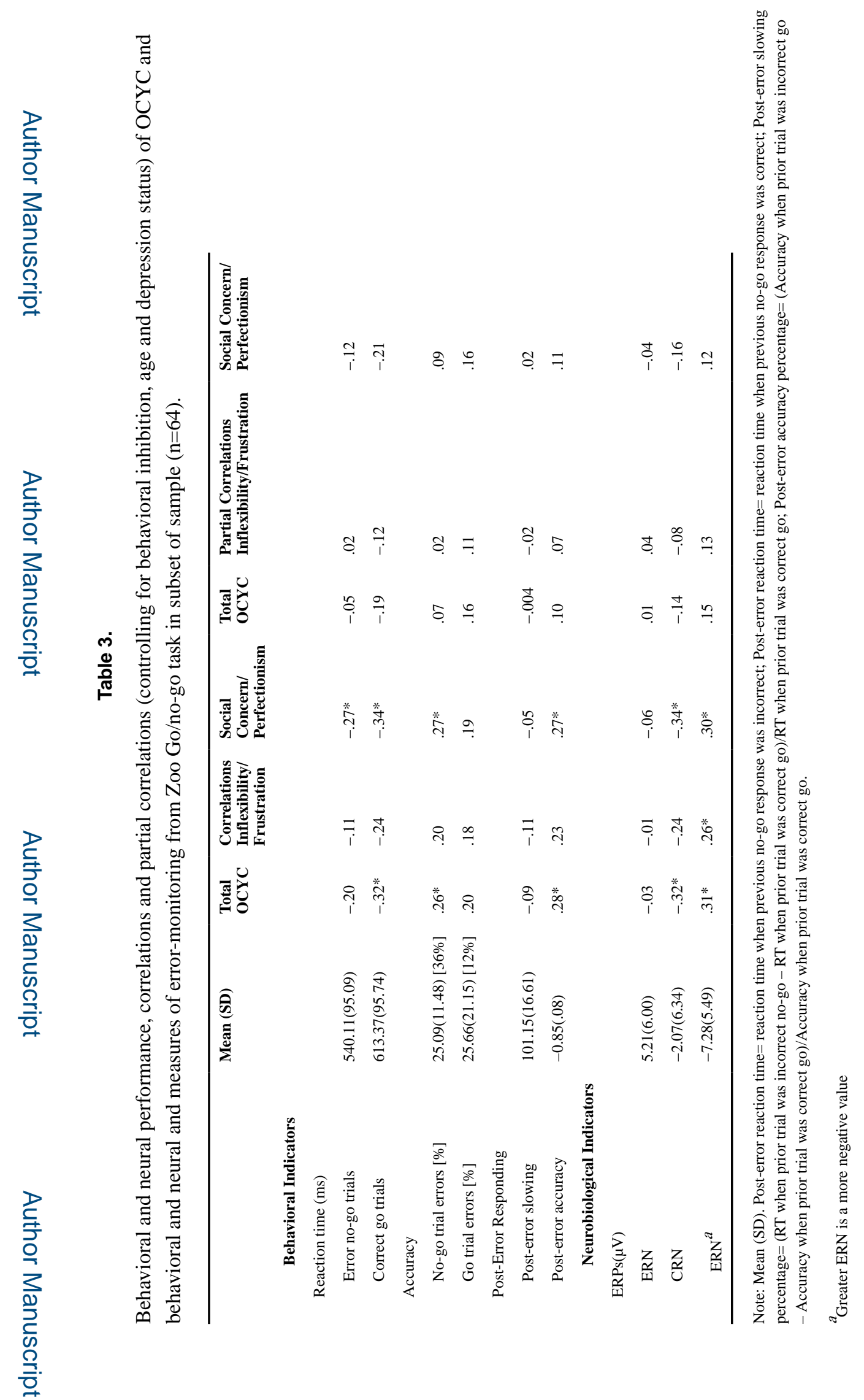

Child Psychiatry Hum Dev. Author manuscript; available in PMC 2021 February 01. 